\title{
RIESGOS ERGONÓMICOS EN LA PRODUCCIÓN DE MUEBLES PARA OFICINA
}

\author{
ERGONOMIC RISKS IN THE PRODUCTION OF OFFICE FURNITURE
}

\author{
Raquel Muñoz Hernández ${ }^{*}$ \\ Saúl Rangel Lara ${ }^{* *}$
}

\begin{abstract}
Resumen: La investigación se desarrolló en una empresa manufacturera de muebles de oficina en el estado de México municipio de Tultitlan de Mariano Escobedo, específicamente en el proceso de empaque, con el objetivo de identificar si existe riesgo biomecánico y su relación con las características antropométricas de los trabajadores, así como su posible relación con daños acumulativos en los trabajadores y enfermedades profesionales. La investigación fue parte de un proyecto general sobre accidentes y ausentismo en el Estado de México. Es una investigación descriptiva de corte transversal. Se evaluaron los puestos de trabajo, de forma inicial, se entrevistó a los operarios y se recopiló información de la accidentalidad en la empresa, posteriormente se observaron los procesos para la evaluación de riesgos con el método Suzzane Rogers. Luego, se llevaron a cabo mediciones antropométricas para la generación de la base de datos con autorización por escrito de los trabajadores. Los resultados muestran que existe riesgo de que los trabajadores adquieran disfunciones traumáticas acumulativas en levantamientos, específicamente a causa de un agarre deficiente. La propuesta fue optimizar el proceso de forma integral, con la implementación de métodos ergonómicos y la readecuación de la estación de trabajo con base en los datos antropométricos obtenidos para un mejor desempeño de las tareas y siguiendo las recomendaciones de la Norma Oficial Mexicana NOM-036-1-STPS-2018.
\end{abstract}

Palabras clave: Ergonomía, repetitividad, puesto de trabajo.

Abstract: The research was developed in an office furniture manufacturing company in the state of Mexico, municipality of Tultitlán de Mariano Escobedo, specifically in the packaging process, with the aim of identifying if there is a biomechanical risk and its relationship with the anthropometric characteristics of the workers, as well as its possible relationship with cumulative damages in workers and occupational diseases. The investigation was part of a general project on accidents and absenteeism in the State of Mexico. It is a descriptive cross-sectional investigation. The jobs were evaluated, initially, the operators were interviewed and information was collected on the accident rate in the company, later the processes for risk assessment were observed with the Suzzane Rogers method. Subsequently, anthropometric measurements were carried out for the generation of the database with the written authorization of the workers. The results show that there is a risk of workers acquiring cumulative traumatic dysfunction in lifting, specifically due to poor grip. The proposal was to optimize the process in an integral way, with the implementation of ergonomic methods and the

"Universidad Politécnica del Valle de México. Municipio de Tultitlán, Estado de México, México. Correo electrónico: raquel.munoz@upvm.edu.mx. Orcid: https://orcid.org/oooo-ooo2-4461-8027. Autor de correspondencia.

**Universidad Politécnica del Valle de México. Municipio de Tultitlán, Estado de México. México. Correo electrónico: industrial@upvm.edu.mx. Orcid: https://orcid.org/oooo-0oo3-1498-340X 
readjustment of the work station based on the anthropometric data obtained for a better performance of the tasks and following the recommendations of the Norma Oficial Mexicana NOM-036-1-STPS2018.

Keywords: Ergonomics, repeatability, workplace.

Recepción: 13.04.2021 / Revisión: 16.04.2021 / Aceptación: 04.05.2021

\section{Introducción}

En los últimos años, el número de empresas en todo el mundo ha incrementado tanto en el sector manufacturero como en el de servicios. Los beneficios son grandes por el empleo y satisfactores que genera; sin embargo también se han encontrado consecuencias no tan halagüeñas debido a que la Organización Internacional del Trabajo (OIT) informa que más de 317 millones de accidentes laborales ocurren cada año y 6.300 personas mueren cada día debido a enfermedades o accidentes laborales (Grandjean, 1983). En la misma fuente, informan que el costo de esta adversidad diaria es enorme y la carga económica de las malas prácticas de seguridad y salud se estiman en un 4 por ciento del producto interno bruto (PIB), global cada año, lo que implica, además de pérdidas humanas, pérdidas financieras (OIT, 2013).

A pesar de que desde el año 2000, la OIT adoptó el programa de salud y seguridad ocupacional y medio ambiente, que tiene como objetivo crear conciencia mundial sobre la magnitud y las consecuencias de los accidentes, las lesiones y las enfermedades relacionadas con el trabajo, así como la identificación de riesgos disergonómicos, las cifras siguen incrementándose (Apud \& Meyer, 2003). Los autores de la presente investigación, consideran que las razones son multifactoriales, sin embargo, cabe hacer notar la importancia de acciones preventivas a través de la divulgación y uso adecuado de la ergonomía como un factor de cambio para la disminución de accidentes.

El término ergonomía proviene de las palabras griegas ergon (trabajo) y nomos (ley, norma o doctrina); la primera referencia a la ergonomía aparece en el libro Compendio de Ergonomía (González, 2007). La International Ergonomics Association ([IEA], 2016), define la ergonomía como "la disciplina científica que se ocupa de la comprensión de la interacción entre los seres humanos y los demás elementos de un sistema, entre los objetivos de la ergonomía está contemplado el logro de la satisfacción en el trabajo, considerando las responsabilidades, actitudes, creencias y valores para el desarrollo personal, así como las diferencias individuales y culturales". Otro enfoque es el que considera la ergonomía como un "elemento educativo del entorno, que facilita la interacción entre el individuo y su ambiente laboral; se traduce en mejor calidad de vida, reducción de posibles accidentes y enfermedades, incremento del bienestar, productividad, cuidado de la salud y medio ambiente" (Universidad Politécnica de Valencia, 2006).

En el presente trabajo, el contexto es una empresa manufacturera, por lo cual se trata de macroergonomía, es decir, está orientado hacia la ergonomía organizacional. Para clarificar el concepto se considera la definición de Hendrick (1991): "La ergonomía 
organizacional o macro ergonomía es el enfoque de los sistemas sociotécnicos para el diseño organizacional y finalmente de los sistemas del trabajo y el diseño de las interfaces relacionadas, hombre-máquina, hombre-entorno y usuario".

La ergonomía como disciplina científica, está relacionada con el desarrollo de conocimientos sobre las capacidades y limitaciones del ser humano en el proceso y desempeño de sus actividades en el contexto de interfaz entre personas, máquinas, sistemas y su entorno. En la actualidad existe un considerable vacío en la aplicación de la ergonomía y solo se aplica en algunos puestos de trabajo del sector manufacturero, sin considerar que es una herramienta de prevención de accidentes y preservación de la salud de todo tipo trabajo para toda persona que realiza una actividad en el desempeño de sus funciones, contribuyendo a mejorar las condiciones de seguridad y salud en el trabajo y por consecuencia en el incremento de la productividad.

Una de las ramas de la ergonomía es la antropometría. En este sentido, Mondelo, Gregori, y Barrau (1999), expresan detalladamente lo que significa antropometría y los aspectos antropométricos que se presentan en la ergonomía geométrica. Estos autores definen la antropometría como "la disciplina que describe las diferencias de las medidas del cuerpo humano, estudia las dimensiones tomando como referencia distintas estructuras anatómicas, y sirve de herramienta a la ergonomía con objeto de adaptar el entorno a las personas".

Como se mencionó anteriormente, el conocimiento y divulgación de la ergonomía ha sido poco difundido y en general no forma parte de la cultura laboral. El mayor problema de este desconocimiento, además de los daños en los trabajadores, es que se desconoce el alcance de las afectaciones debido a que no se llevan registros documentados centralizados, ni se hacen seguimientos de las cifras reales de lesiones, accidentes y enfermedades profesionales por factores disergonómicos en los centros de trabajo. Además, los datos registrados en el Seguro Social no son confiables, debido a que no todas las empresas tienen a sus empleados dados de alta con dicha prestación, sin embargo han servido como referencia para el presente estudio.

Esta investigación se genera a partir de la necesidad de conocer las causas de la recurrencia de quejas de los trabajadores con respecto a dolor articular, de espalda e incapacidades frecuentes, en el área de empaque de una planta de producción de muebles de oficina ubicada en el municipio de Tultitlán en el Estado de México.

Con base en lo anterior, en este proyecto se investigan los procedimientos en los levantamientos así como los perfiles antropométricos de los trabajadores de la zona norte del Estado de México, con el fin de elaborar un análisis para determinar si existe relación entre la accidentalidad y las características antropométricas de los trabajadores y documentar los resultados. Al buscar estudios previos con temas relacionados al problema planteado en la presente investigación, se encontraron pocos, de los cuales solo se mencionan dos por el enfoque tan radical entre ellos y no se llevaron a cabo en México.

En el primero se estudian funciones en plantas de la industria manufacturera, mencionando que los riesgos son inherentes para los trabajadores y que los efectos se percibirán en un mediano o largo plazo. De acuerdo a los estudios ergonómicos realizados 
en el sector metalmecánico, sobre la carga física en trabajadores del área de acabados en una industria metalmecánica en el departamento de Risaralda, se encontró de que los seis puestos estudiados presentaron riesgo medio, lo cual no es concluyente, además de no estar relacionado con la antropometría, y los resultados muestran cierto grado de subjetividad (Galvis et al., 2015).

El segundo caso es el método propuesto por Move Human-Forces desarrollado por el equipo de investigación del Prof. Marín de la Universidad de Zaragoza, y dirigido a evaluar el riesgo musculoesquelético derivado de la realización de tareas repetitivas con alta frecuencia, característico de entornos de producción con ciclos de fabricación cortos. Se fundamenta en relacionar la antropometría y el riesgo de adquirir una lesión. Todo lo anterior resulta muy atractivo y los resultados son más objetivos por tratarse de cálculos biomecánicos y no depender del criterio e interpretación del analista, la desventaja es que el equipo es costosos e invasivo. (Marín-Zurdo et al., 2013).

Ambos estudios analizan el tema del presente con métodos diferentes y para el caso de México, primeramente se observa la parte legal y normativa establecida por la Secretaría de Trabajo y Previsión Social (STPS), quien establece los mecanismos (leyes, reglamentos, normas, entre otros), a través de los cuales se rigen las relaciones laborales de salud y seguridad, así como mantenerlas actualizadas; donde la Norma Oficial Mexicana NOM-o361-STPS-2018, establece que los factores de riesgo ergonómico pueden derivar en esfuerzo físico, movimientos repetitivos o posturas forzadas en el trabajo realizado, con la consecuente fatiga, errores, accidentes y enfermedades laborales derivadas del diseño de las instalaciones, maquinaria, equipos, herramientas o puesto de trabajo. Estos incluyen, el manejo manual de cargas, sobreesfuerzo físico, movimientos repetitivos y posturas forzadas. En la primera parte del documento, se establecen los criterios relacionados con la manipulación manual de cargas superiores a $3 \mathrm{~kg}$. Por este motivo, se excluyen los centros donde se manipulen cargas menores. Uno de los criterios más importantes que establece la norma es la relación entre el peso máximo que puede llevar una persona determinado por su sexo y edad de acuerdo a lo publicado en el Diario Oficial de la Federación de enero del 2020 ([DOF], 2020).

Uno de los factores que se identificaron desde un principio fue que, por su origen, las dimensiones y diseño de las máquinas suelen ser muy diferentes a las características antropométricas de los usuarios y esto ha provocado que los operadores tengan que adaptarse a su puesto de trabajo en equipos y maquinarias, con el riesgo de adquirir alguna disfunción traumática acumulativa (DTA). Por otra parte, al observar las actividades y desempeño de los trabajadores se pudo ver que siguen ejecutando levantamientos manuales que sobrepasan los límites en kilogramos permisibles a pesar de que las autoridades laborales les han aclarado que la Norma Oficial Mexicana NOM-036-1-STPS-2018 no establece requisitos de edad específicos para la contratación o permanencia del personal, solo establece la masa máxima que un trabajador puede levantar o descender en función de su edad y sexo.

Cabe mencionar, que al platicar directamente con los trabajadores, algunos de ellos, manifestaron temor de perder su empleo, debido a que se ha tergiversado la interpretación de la norma creando la idea de que cuando un trabajador cumpla 45 años y ya no pueda realizar el manejo manual de cargas hasta el límite máximo permitido será despedido; lo cual 
es incorrecto; por el contrario, la norma impone al empresario la obligación de vigilar de forma especial la salud del colaborador para que continúe prestando sus servicios a la empresa de forma segura que preserve su salud.

Además de los criterios para el análisis de factores de riesgo, la Norma Oficial Mexicana NOM-036-1-STPS-2018 establece medidas de prevención y control para cuidar la salud de los trabajadores. Asimismo, indica acciones de seguridad para los trabajadores que realizan posturas forzadas derivadas del manejo de la carga. Con su eventual cumplimiento, los centros de trabajo contarán con condiciones más seguras para los trabajadores.

Con base en las inquietudes del personal en lo referente a la legalidad y a la preocupación de los empresarios en lo referente a las recurrentes incapacidades e inasistencias de los trabajadores, se llevó a cabo una investigación, observacional, descriptiva y transversal, para determinar el perfil antropométrico de la población trabajadora, los procesos productivos en lo referente a movimientos repetitivos con base en la Norma Oficial Mexicana NOM-036-1-STPS-2018.

El objetivo es evaluar el posible riesgo de adquirir disfunciones traumático acumulativas (DTA's) al identificar si existe relación entre la accidentabilidad y los procesos de levantamientos, y entre la accidentabilidad y la antropometría de los trabajadores.

\section{Materiales y métodos}

La muestra se integró por participantes de forma voluntaria, a quienes antes de realizar las mediciones se les explicó el procedimiento y los objetivos del estudio, y así mismo se les hizo entrega de una hoja de consentimiento del uso de sus datos, y salvaguarda de los mismos, los cuales son utilizados única y exclusivamente para la investigación. Se les proporcionó la información necesaria sobre el procedimiento, los riesgos, beneficios, sus derechos y la confidencialidad del estudio. Finalmente, el documento se firmó por el voluntario, el responsable del proyecto y un testigo.

Para la evaluación ergonómica participaron 16 personas, hombres entre 19 y 65 años, la muestra se formó únicamente por personal del género masculino debido a que en la sección estudiada no laboran mujeres y se organizaron en grupos por edades. Se llevó a cabo en la estación de empaque y emplayado de muebles.

Se aplicó el método Suzanne Rodgers, que analiza siete regiones del cuerpo para la evaluación de la fatiga acumulada con actividades repetitivas que pueden causar lesiones musculoesqueléticas. Este método es más apropiado para evaluar el riesgo por acumulación de fatiga en tareas ejecutadas durante una hora o más, y en donde se encuentran presentes malas posturas y esfuerzos repetitivos (Cornejo \& Quiñónez, 2007). Los esfuerzos se clasifican por la duración y frecuencia como se muestra en la tabla 1. 
Tabla 1. Valoración de la duración y frecuencia.

\begin{tabular}{|c|c|c|}
\hline Clasificación & $\begin{array}{c}\text { Duración para un nivel de esfuerzo } \\
\text { específico }\end{array}$ & Esfuerzos por minuto \\
\hline 1 & $<6$ segundos & $<1$ por minuto \\
\hline 2 & Entre 6 y 20 segundos & 1 a 5 por minuto \\
\hline 3 & $>20$ segundos & $>5$ y hasta 15 por minuto \\
\hline
\end{tabular}

Posteriormente, se realizó un estudio sobre el área con la información económica básica del Instituto Nacional de Estadística y Geografía (INEGI) que es uno de los Órganos constitucionales autónomos de México con gestión, personalidad jurídica y patrimonio propios, responsable de normar y coordinar el Sistema Nacional de Información Estadística y Geografía.

Luego, se obtuvieron las autorizaciones correspondientes de la institución y se llevó a cabo el reclutamiento de los integrantes de los equipos para apoyo en los estudios antropométricos dentro del mismo personal, y fueron capacitados en antropometría para tomar las medidas.

Previamente, se realizó una revisión bibliográfica con el objetivo de conocer qué dimensiones antropométricas son las más utilizadas por los diseñadores, relacionados con la antropometría para el diseño de mobiliario, herramientas y puestos de trabajo. Una vez realizado lo anterior, se inició la toma de las medidas antropométricas de la muestra en los lugares de trabajo con el equipo antropométrico y los formatos para el registro de datos. El formulario de consentimiento informado se revisó con cada voluntario, utilizando la técnica antropométrica de Hertzberg (1968). Los trabajadores firmaron la documentación correspondiente y vistieron ropa adecuada para la ocasión; es decir, ceñida al cuerpo, de ser posible de licra y de manga corta. Las mediciones las realizaron personal de género masculino y en todo momento se mantuvo un ambiente de cordialidad, respeto y confidencialidad.

Se realizaron mediciones antropométricas de 42 puntos que se especifican y numeran en la tabla 2, para la generación de la base de datos. 
generalmente referidas al menos a un punto de referencia de una altura definida apoyándose en la planta de un pie o girando el tronco y la cintura.

Se considera además la inclinación del cuello, giro de las manos, abducción de los dedos por tratarse de empresa manufacturera y ser el movimiento más recurrente del trabajo manual; así mismo la longitud y ancho de la mano derecha e izquierda incluyendo el diámetro de agarre que es tan importante para levantamiento y sujeción.

En lo referente a las mediciones antropométricas se obtuvieron los percentiles 5, 50 y 95. Se realizó un análisis de contingencia, pruebas de normalidad, medidas de tendencia central, dispersión y frecuencia esperada de coincidencia entre variables, con el fin de identificar las partes del cuerpo en el uso común de equipo y herramienta.

Se verificó de manera directa la forma en que los operarios realizan sus actividades y levantamientos de carga. Se utilizaron las ilustraciones de las siguientes figuras para guiar la evaluación, tomando como referencia lo establecido en la Norma Oficial Mexicana NOM036-1-STPS-2018. Se observó la posición de las manos del trabajador al comienzo, y a medida que avanza la operación, se fue observando el peor escenario.

Figura 1. Distancia horizontal entre manos y espalda baja (DOF, 2020).

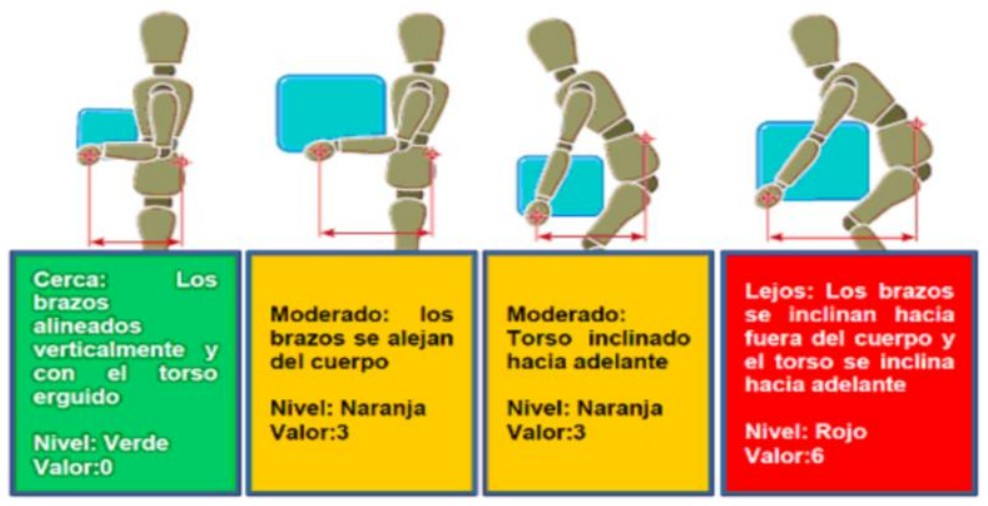

Figura 2. Región de levantamiento vertical (DOF, 2020).
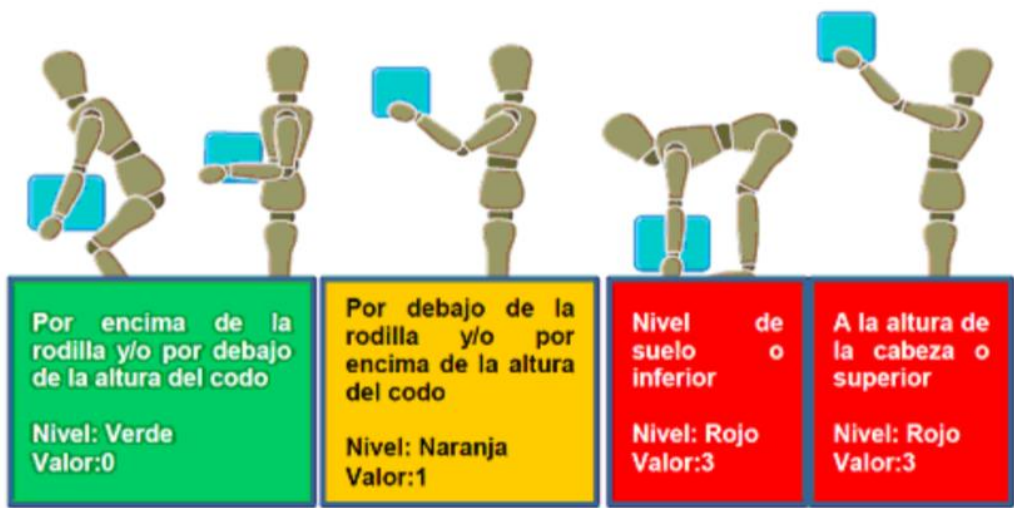
Figura 3. Torsión del torso y flexión lateral (DOF, 2020).

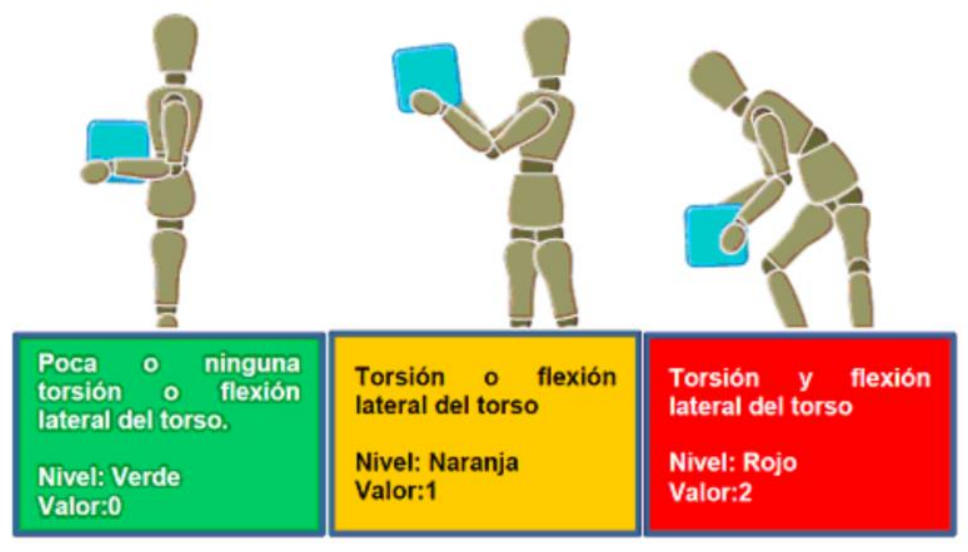

Al finalizar las mediciones se realizó la captura, revisión, corrección y procesamiento de los datos con el software IBM SPSS para Windows en versión 15.0.

\section{Resultados y discusión}

En la figura 4 se muestran los resultados de la aplicación del método Suzanne Rodgers. 
Figura 4. Resultados método Suzanne Rodgers.

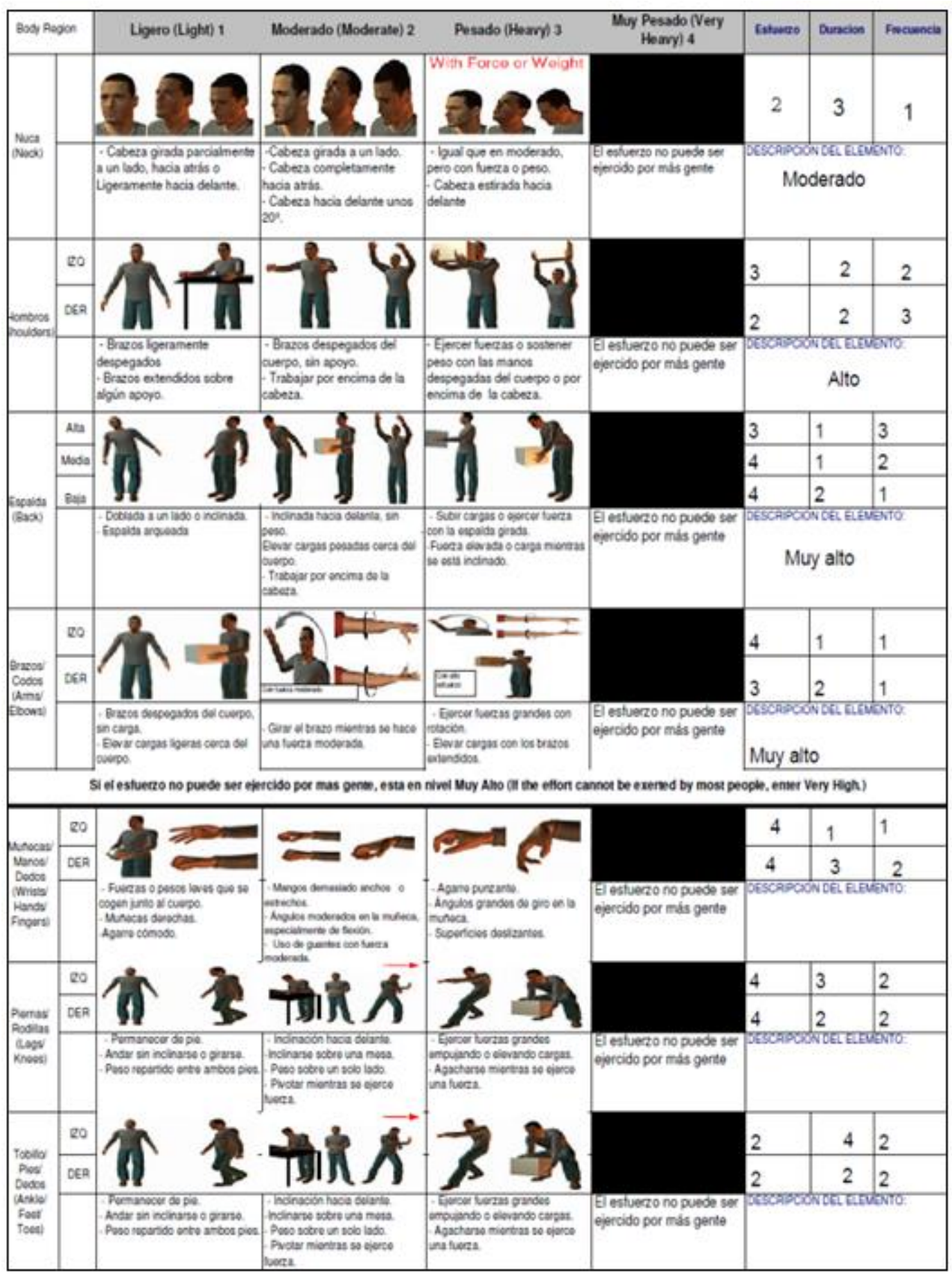

Para realizar este análisis es vital una tabla llamada condiciones de severidad. La severidad es 4 en casi todos los casos, lo significa que existe un potencial de riesgo elevado para la salud del trabajador que ejerce esta labor.

En la tabla 3 se observan los resultados sobre la prioridad de cambio. 
Tabla 3. Resultados prioridad de cambio.

\begin{tabular}{|l|c|c|c|}
\hline \multicolumn{1}{|c|}{ Clasificación } & Nivel de esfuerzo & Duración & Frecuencia \\
\hline Cuello & 2 & 3 & 1 \\
\hline Brazos, codos & 4 & 2 & 1 \\
\hline Hombros & 3 & 2 & 2 \\
\hline Manos, dedos & 2 & 2 & 3 \\
\hline Piernas, rodillas & 3 & 1 & 2 \\
\hline Espalda & 4 & 1 & 2 \\
\hline Muñeca & 4 & 1 & 1 \\
\hline Codos & 4 & 3 & 2 \\
\hline Piernas & 4 & 3 & 2 \\
\hline Rodilla & 4 & 2 & 2 \\
\hline
\end{tabular}

En cuanto a las mediciones antropométricas, las dimensiones lineales de los 16 trabajadores se muestran en la tabla 4, y las dimensiones angulares en la tabla 5 .

Tabla 4. Dimensiones lineales en $\mathrm{cm}$, edad y peso.

\begin{tabular}{|c|c|c|c|c|c|c|c|c|c|c|c|c|c|c|c|c|c|}
\hline \multirow{2}{*}{ No. } & \multirow{2}{*}{ Descripción } & \multicolumn{16}{|c|}{ Trabajador } \\
\hline & & $\begin{array}{l}\mathbf{T} \\
\mathbf{1}\end{array}$ & $\begin{array}{l}\mathbf{T} \\
\mathbf{2}\end{array}$ & $\begin{array}{l}\mathbf{T} \\
\mathbf{3}\end{array}$ & $\begin{array}{l}T \\
4\end{array}$ & $\begin{array}{l}\mathbf{T} \\
5\end{array}$ & $\begin{array}{l}T \\
6\end{array}$ & $\begin{array}{l}\mathbf{T} \\
7\end{array}$ & $\begin{array}{l}\mathbf{T} \\
\mathbf{8}\end{array}$ & $\begin{array}{l}\mathbf{T} \\
\mathbf{9}\end{array}$ & $\begin{array}{l}\mathbf{T} \\
\mathbf{1 0}\end{array}$ & $\begin{array}{l}\mathbf{T} \\
\mathbf{1 1}\end{array}$ & $\begin{array}{l}\mathbf{T} \\
12\end{array}$ & $\begin{array}{l}\mathbf{T} \\
13\end{array}$ & $\begin{array}{l}T \\
14\end{array}$ & $\begin{array}{l}\mathbf{T} \\
\mathbf{1 5}\end{array}$ & $\begin{array}{l}T \\
16\end{array}$ \\
\hline 1 & Estatura & $\begin{array}{r}17 \\
2 \\
\end{array}$ & $\begin{array}{c}17 \\
9 \\
\end{array}$ & $\begin{array}{c}18 \\
0\end{array}$ & $\begin{array}{c}17 \\
6 \\
\end{array}$ & $\begin{array}{c}17 \\
5\end{array}$ & $\begin{array}{c}16 \\
4\end{array}$ & $\begin{array}{c}17 \\
2 \\
\end{array}$ & $\begin{array}{c}15 \\
6 \\
\end{array}$ & $\begin{array}{c}16 \\
1 \\
\end{array}$ & 172 & $\begin{array}{c}18 \\
4\end{array}$ & 168 & 184 & 169 & 178 & 165 \\
\hline 2 & Altura al hombro & $\begin{array}{c}14 \\
1\end{array}$ & $\begin{array}{c}15 \\
0\end{array}$ & $\begin{array}{c}15 \\
0\end{array}$ & $\begin{array}{c}14 \\
6\end{array}$ & $\begin{array}{c}14 \\
8\end{array}$ & $\begin{array}{c}14 \\
6\end{array}$ & $\begin{array}{c}14 \\
9\end{array}$ & $\begin{array}{c}15 \\
8\end{array}$ & $\begin{array}{c}14 \\
3\end{array}$ & 151 & $\begin{array}{c}16 \\
0\end{array}$ & 142 & 159 & 145 & 155 & 138 \\
\hline 3 & Altura al codo & $\begin{array}{c}10 \\
6 \\
\end{array}$ & $\begin{array}{l}8 \\
4 \\
\end{array}$ & $\begin{array}{c}10 \\
9 \\
\end{array}$ & $\begin{array}{c}10 \\
6 \\
\end{array}$ & $\begin{array}{c}10 \\
7 \\
\end{array}$ & $\begin{array}{c}10 \\
1\end{array}$ & $\begin{array}{c}10 \\
5 \\
\end{array}$ & $\begin{array}{c}12 \\
0\end{array}$ & $\begin{array}{c}10 \\
5 \\
\end{array}$ & 110 & $\begin{array}{c}12 \\
2 \\
\end{array}$ & 98 & 114 & 104 & 112 & 100 \\
\hline 4 & Altura brazo estirado & $\begin{array}{c}20 \\
2\end{array}$ & $\begin{array}{l}21 \\
7\end{array}$ & $\begin{array}{l}2 \\
2 \\
0 \\
\end{array}$ & $\begin{array}{c}21 \\
6\end{array}$ & $\begin{array}{c}21 \\
7\end{array}$ & $\begin{array}{l}2 \\
0 \\
0 \\
\end{array}$ & $\begin{array}{l}2 \\
0 \\
4 \\
\end{array}$ & $\begin{array}{l}21 \\
8\end{array}$ & $\begin{array}{c}14 \\
7\end{array}$ & 203 & $\begin{array}{c}21 \\
6\end{array}$ & 200 & 222 & 201 & 215 & 198 \\
\hline 5 & $\begin{array}{l}\text { Largo desde la vertical } \\
\text { al puño }\end{array}$ & 78 & $\begin{array}{l}8 \\
2 \\
\end{array}$ & 73 & 78 & $\begin{array}{l}7 \\
0\end{array}$ & 76 & 75 & 81 & 67 & 72 & 76 & 74 & 80 & 75 & 76 & 74 \\
\hline 6 & $\begin{array}{l}\text { Largo desde la vertical } \\
\text { al pecho }\end{array}$ & 25 & $\begin{array}{l}2 \\
6\end{array}$ & $\begin{array}{l}2 \\
4\end{array}$ & $\begin{array}{l}4 \\
0\end{array}$ & $\begin{array}{l}2 \\
4\end{array}$ & $\begin{array}{l}2 \\
0\end{array}$ & $\begin{array}{l}2 \\
4\end{array}$ & 25 & 23 & 22 & 24 & 16 & 20 & 21 & 23 & 20 \\
\hline 7 & Anchura de hombros & 45 & $\begin{array}{l}4 \\
3\end{array}$ & $\begin{array}{l}4 \\
3\end{array}$ & 59 & $\begin{array}{l}4 \\
2\end{array}$ & 46 & $\begin{array}{l}4 \\
4\end{array}$ & 45 & $\begin{array}{l}4 \\
3\end{array}$ & 43 & 43 & 42 & 45 & 48 & 46 & 46 \\
\hline 8 & Ancho de clavículas & 32 & $\begin{array}{l}3 \\
8\end{array}$ & $\begin{array}{l}3 \\
3\end{array}$ & $\begin{array}{l}4 \\
0\end{array}$ & $\begin{array}{l}3 \\
2\end{array}$ & 35 & $\begin{array}{l}3 \\
6\end{array}$ & 36 & $\begin{array}{l}2 \\
9\end{array}$ & 26 & 20 & 36 & 38 & 36 & 41 & 39 \\
\hline 9 & $\begin{array}{l}\text { Altura del glúteo al } \\
\text { arco de media espalda }\end{array}$ & 25 & 21 & $\begin{array}{l}2 \\
3 \\
\end{array}$ & 25 & $\begin{array}{l}2 \\
4 \\
\end{array}$ & 25 & $\begin{array}{l}3 \\
4 \\
\end{array}$ & 29 & 27 & 26 & 27 & 56 & 60 & 60 & 56 & 58 \\
\hline 10 & $\begin{array}{l}\text { Altura del piso a la } \\
\text { mitad de la espinilla }\end{array}$ & 46 & $\begin{array}{l}3 \\
8\end{array}$ & 45 & $\begin{array}{l}4 \\
3\end{array}$ & $\begin{array}{l}4 \\
5\end{array}$ & 39 & $\begin{array}{l}4 \\
2\end{array}$ & $\begin{array}{l}4 \\
8\end{array}$ & $\begin{array}{l}4 \\
3\end{array}$ & 43 & 47 & 41 & 44 & 40 & 46 & 40 \\
\hline 11 & $\begin{array}{l}\text { Largo desde el glúteo a } \\
\text { la rodilla }\end{array}$ & 59 & 53 & 56 & 57 & 55 & $\begin{array}{l}4 \\
8\end{array}$ & $\begin{array}{l}6 \\
4\end{array}$ & 57 & 55 & 53 & 59 & 42 & 48 & 44 & 46 & 44 \\
\hline 12 & Anchura de hombros & 45 & 51 & $\begin{array}{l}4 \\
2\end{array}$ & $\begin{array}{l}6 \\
0\end{array}$ & $\begin{array}{l}4 \\
4 \\
\end{array}$ & 47 & 41 & 45 & $\begin{array}{l}4 \\
0 \\
\end{array}$ & 47 & 49 & 14 & 15 & 14 & 17 & 15 \\
\hline 13 & $\begin{array}{l}\text { Largo de la mano al } \\
\text { dedo medio }\end{array}$ & 16 & 19 & 18 & 15 & 17 & 16 & 18 & 18 & 17 & 17 & 14 & 19 & 19 & 20 & 22 & 18 \\
\hline 14 & Anchura de mano & 11 & 10 & 11 & 12 & 10 & 10 & 12 & 10 & 11 & 11 & 10 & 17 & 17 & 14 & 17 & 19 \\
\hline 15 & Anchura de muñeca & 6 & 8 & 7 & 10 & 8 & 9 & 8 & 7 & 6 & 7 & 6 & 10 & 10 & 10 & 11 & 11 \\
\hline 16 & Diámetro de agarre & 4 & 4 & 3 & 3 & 3 & 4 & 4 & 4 & 3 & 5 & 6 & 6 & 7 & 7 & 6 & 6 \\
\hline
\end{tabular}




\begin{tabular}{|c|c|c|c|c|c|c|c|c|c|c|c|c|c|c|c|c|c|}
\hline 17 & Largo del pie & 26 & 27 & 27 & $\begin{array}{l}2 \\
8 \\
\end{array}$ & $\begin{array}{l}2 \\
6 \\
\end{array}$ & 25 & $\begin{array}{l}2 \\
8 \\
\end{array}$ & $\begin{array}{l}3 \\
0 \\
\end{array}$ & 25 & 26 & 29 & 4 & 5 & 3 & 4 & 3 \\
\hline 18 & Anchura del pie & 10 & 11 & 10 & 11 & 9 & 10 & 10 & 10 & 9 & 10 & 11 & 27 & 29 & 27 & 28 & 26 \\
\hline 19 & Altura en cuclillas & $\begin{array}{c}10 \\
9\end{array}$ & $\begin{array}{c}12 \\
9\end{array}$ & $\begin{array}{c}12 \\
0\end{array}$ & $\begin{array}{c}12 \\
9\end{array}$ & $\begin{array}{c}11 \\
8\end{array}$ & $\begin{array}{c}11 \\
5\end{array}$ & $\begin{array}{c}13 \\
0\end{array}$ & $\begin{array}{c}13 \\
7\end{array}$ & $\begin{array}{c}12 \\
3\end{array}$ & 122 & 121 & 116 & 123 & 124 & 125 & 124 \\
\hline 20 & $\begin{array}{l}\text { Altura del piso a brazo } \\
\text { extendido }\end{array}$ & $\begin{array}{c}14 \\
5 \\
\end{array}$ & $\begin{array}{c}15 \\
4 \\
\end{array}$ & $\begin{array}{c}14 \\
9 \\
\end{array}$ & $\begin{array}{c}14 \\
4 \\
\end{array}$ & $\begin{array}{c}14 \\
7 \\
\end{array}$ & $\begin{array}{c}14 \\
7 \\
\end{array}$ & $\begin{array}{c}14 \\
8\end{array}$ & $\begin{array}{c}14 \\
7 \\
\end{array}$ & $\begin{array}{c}14 \\
5 \\
\end{array}$ & 148 & $\begin{array}{c}14 \\
6\end{array}$ & 137 & 144 & 142 & 143 & 136 \\
\hline 21 & Edad & 20 & $\begin{array}{l}2 \\
6\end{array}$ & $\begin{array}{l}2 \\
O\end{array}$ & 33 & $\begin{array}{l}4 \\
5\end{array}$ & 25 & 56 & 43 & 27 & 44 & 22 & 36 & 19 & 19 & 23 & 29 \\
\hline 22 & Peso en kg & 72 & $\begin{array}{l}8 \\
8\end{array}$ & $\begin{array}{l}9 \\
O\end{array}$ & 79 & $\begin{array}{l}8 \\
8\end{array}$ & 72 & 87 & 70 & $\begin{array}{l}6 \\
8\end{array}$ & 84 & 85 & 72 & 80 & 67 & 80 & 70 \\
\hline
\end{tabular}

Tabla 5. Dimensiones angulares.

\begin{tabular}{|c|c|c|c|c|c|c|c|c|c|c|c|c|c|c|c|c|}
\hline Descripción & \multicolumn{16}{|c|}{ Trabajador } \\
\hline $\begin{array}{c}\text { Ángulos mano } \\
\text { derecha }\end{array}$ & $\begin{array}{l}\mathbf{T} \\
\mathbf{1}\end{array}$ & $\begin{array}{l}\mathbf{T} \\
\mathbf{2}\end{array}$ & $\begin{array}{l}\mathbf{T} \\
\mathbf{3}\end{array}$ & $\begin{array}{l}T \\
4\end{array}$ & $\begin{array}{l}\mathbf{T} \\
\mathbf{5}\end{array}$ & $\begin{array}{l}T \\
6\end{array}$ & $\begin{array}{l}\mathbf{T} \\
7\end{array}$ & $\begin{array}{l}\mathbf{T} \\
\mathbf{8}\end{array}$ & $\begin{array}{l}\mathbf{T} \\
\mathbf{9}\end{array}$ & $\begin{array}{c}\mathbf{T} \\
\mathbf{1 0}\end{array}$ & $\begin{array}{c}\mathbf{T} \\
\mathbf{1 1}\end{array}$ & $\begin{array}{r}\mathbf{T} \\
\mathbf{1 2}\end{array}$ & $\begin{array}{c}\mathbf{T} \\
\mathbf{1 3}\end{array}$ & $\begin{array}{c}\mathbf{T} \\
14\end{array}$ & $\begin{array}{r}\mathbf{T} \\
\mathbf{1 5}\end{array}$ & $\begin{array}{r}\mathrm{T} \\
16\end{array}$ \\
\hline $\begin{array}{l}\mathrm{MD}_{-} \\
\text {Dedo pulgar - dedo índice }\end{array}$ & 88 & 92 & 88 & 84 & 95 & 87 & 83 & 83 & 60 & 79 & 89 & 92 & 88 & 91 & 87 & 86 \\
\hline $\begin{array}{l}\mathrm{MD}_{-} \\
\text {Dedo índice - dedo medio }\end{array}$ & 46 & 51 & 58 & 58 & 58 & 44 & 58 & 73 & 55 & 62 & 45 & 50 & 45 & 47 & 44 & 44 \\
\hline $\begin{array}{l}\mathrm{MD}_{-} \\
\text {Dedo medio - dedo anular }\end{array}$ & 34 & 40 & 62 & 46 & 64 & 36 & 61 & 60 & 46 & 57 & 36 & 38 & 35 & 38 & 35 & 34 \\
\hline $\begin{array}{l}\text { MD_- } \\
\text { Dedo anular - dedo meñique }\end{array}$ & 32 & 39 & 40 & 59 & 53 & 33 & 63 & 69 & 70 & 60 & 34 & 39 & 32 & 37 & 34 & 31 \\
\hline $\begin{array}{l}\text { MD } \\
\text { Abducción }\end{array}$ & 94 & 98 & 74 & 72 & 97 & 93 & 94 & 91 & 94 & 96 & 93 & 97 & 93 & 95 & 92 & 92 \\
\hline $\begin{array}{l}\text { MD } \\
\text { Supinación }\end{array}$ & 35 & 43 & 67 & 64 & 63 & 60 & 64 & 53 & 54 & 38 & 43 & 39 & 42 & 36 & 45 & 55 \\
\hline $\begin{array}{l}\text { MD_- } \\
\text { Lateral izquierdo }\end{array}$ & 60 & 72 & 60 & 88 & 63 & 75 & $\begin{array}{c}12 \\
8 \\
\end{array}$ & 119 & 45 & 85 & 68 & 65 & 78 & 78 & 63 & 39 \\
\hline $\begin{array}{l}\text { MD_} \\
\text { Lateral derecho }\end{array}$ & 56 & 67 & 58 & 38 & $\begin{array}{c}10 \\
4\end{array}$ & 67 & 58 & 44 & 53 & 65 & 54 & 55 & 55 & 54 & 62 & 72 \\
\hline $\begin{array}{c}\text { Ángulos mano } \\
\text { izquierda }\end{array}$ & $\begin{array}{l}\mathbf{T} \\
\mathbf{1}\end{array}$ & $\begin{array}{l}\mathbf{T} \\
\mathbf{2}\end{array}$ & $\mathbf{T}$ & $\mathbf{T}$ & $\begin{array}{l}\mathbf{T} \\
\mathbf{5}\end{array}$ & $\mathbf{T}$ & $\begin{array}{l}\mathbf{T} \\
7\end{array}$ & $\begin{array}{l}\mathbf{T} \\
\mathbf{8}\end{array}$ & $\begin{array}{l}\mathbf{T} \\
\mathbf{9}\end{array}$ & $\begin{array}{c}\mathbf{T} \\
\mathbf{1 0}\end{array}$ & $\mathbf{T}$ & $\begin{array}{c}\mathbf{T} \\
\mathbf{1 2}\end{array}$ & $\begin{array}{c}\mathbf{T} \\
\mathbf{1 3}\end{array}$ & $\begin{array}{c}\mathrm{T} \\
14\end{array}$ & $\begin{array}{r}\mathbf{T} \\
\mathbf{1 5}\end{array}$ & $\begin{array}{c}\mathrm{T} \\
16\end{array}$ \\
\hline $\begin{array}{l}\text { MI } \\
\text { Dedo pulgar - dedo índice }\end{array}$ & 87 & 92 & 74 & 83 & 91 & 87 & 88 & 68 & 81 & 78 & 88 & 91 & 86 & 94 & 89 & 82 \\
\hline $\begin{array}{l}\text { MI } \\
\text { Dedo índice - dedo medio }\end{array}$ & 48 & 50 & 50 & 51 & 61 & 47 & 63 & 59 & 58 & 62 & 47 & 48 & 44 & 42 & 43 & 46 \\
\hline $\begin{array}{l}\text { MI } \\
\text { Dedo medio - dedo anular }\end{array}$ & 38 & 42 & 52 & 56 & 57 & 32 & 52 & 56 & 54 & 53 & 32 & 38 & 34 & 37 & 37 & 39 \\
\hline $\begin{array}{l}\text { MI } \\
\text { Dedo anular - dedo meñique }\end{array}$ & 37 & 40 & 47 & 58 & 63 & 37 & 55 & 56 & 52 & 61 & 38 & 34 & 36 & 34 & 39 & 34 \\
\hline $\begin{array}{l}\text { MI } \\
\text { Abducción }\end{array}$ & 94 & 97 & 80 & 70 & 95 & 90 & 83 & 85 & 81 & 57 & 74 & 95 & 94 & 92 & 94 & 90 \\
\hline $\begin{array}{l}\text { MI_ } \\
\text { Supinación }\end{array}$ & 48 & 44 & 54 & 60 & 74 & 42 & $\begin{array}{c}12 \\
6 \\
\end{array}$ & $\begin{array}{r}13 \\
2 \\
\end{array}$ & 75 & 94 & 42 & 43 & 44 & 46 & 47 & 54 \\
\hline $\begin{array}{l}\text { MI_- } \\
\text { Lateral izquierdo }\end{array}$ & 60 & 72 & 79 & 73 & 74 & 75 & $\begin{array}{c}12 \\
0\end{array}$ & $\begin{array}{c}13 \\
4\end{array}$ & 61 & 63 & 64 & 65 & 78 & 76 & 63 & 65 \\
\hline $\begin{array}{l}\text { MI_- } \\
\text { Lateral derecho }\end{array}$ & 58 & 68 & 55 & 55 & 54 & 69 & 57 & 42 & 52 & 62 & 52 & 52 & 55 & 54 & 62 & 67 \\
\hline
\end{tabular}

En el periodo del estudio se incapacitaron 4 trabajadores durante 3 días y los percentiles cambiaron como se observa en la tabla 6 . 
Tabla 6. Percentiles de 12 trabajadores.

\begin{tabular}{|c|c|c|c|c|c|c|c|c|c|c|c|c|c|c|c|c|c|}
\hline No. & Descripción & $\begin{array}{l}\mathbf{T} \\
\mathbf{2}\end{array}$ & $\begin{array}{l}\mathbf{T} \\
\mathbf{3}\end{array}$ & $\begin{array}{l}T \\
4\end{array}$ & $\begin{array}{l}T \\
6\end{array}$ & $\begin{array}{l}\mathbf{T} \\
7\end{array}$ & $\begin{array}{l}T \\
8\end{array}$ & $\begin{array}{l}\mathbf{T} \\
\mathbf{9}\end{array}$ & $\begin{array}{c}\mathbf{T} \\
\mathbf{1 1}\end{array}$ & $\begin{array}{c}\mathbf{T} \\
\mathbf{1 2}\end{array}$ & $\begin{array}{c}\mathbf{T} \\
\mathbf{1 4}\end{array}$ & $\begin{array}{c}\mathbf{T} \\
\mathbf{1 5}\end{array}$ & $\underset{16}{T}$ & $\boldsymbol{\mu}$ & $\boldsymbol{o}^{\prime}$ & $\mathbf{P}_{5}$ & P95 \\
\hline 1 & Estatura & $\begin{array}{c}17 \\
9\end{array}$ & $\begin{array}{c}18 \\
0\end{array}$ & $\begin{array}{c}17 \\
6\end{array}$ & $\begin{array}{c}16 \\
4\end{array}$ & $\begin{array}{c}17 \\
2\end{array}$ & $\begin{array}{c}15 \\
6\end{array}$ & $\begin{array}{c}16 \\
1\end{array}$ & $\begin{array}{c}18 \\
4\end{array}$ & $\begin{array}{c}16 \\
8\end{array}$ & 169 & 178 & 165 & $\begin{array}{c}17 \\
1\end{array}$ & 9 & 157 & 185 \\
\hline 2 & Altura al hombro & $\begin{array}{c}15 \\
0\end{array}$ & $\begin{array}{c}15 \\
0\end{array}$ & $\begin{array}{c}14 \\
6\end{array}$ & $\begin{array}{c}14 \\
6\end{array}$ & $\begin{array}{c}14 \\
9\end{array}$ & $\begin{array}{c}15 \\
8\end{array}$ & $\begin{array}{c}14 \\
3\end{array}$ & $\begin{array}{c}16 \\
0\end{array}$ & 142 & 145 & 155 & 138 & $\begin{array}{c}14 \\
9\end{array}$ & 7 & 138 & 159 \\
\hline 3 & Altura al codo & 84 & $\begin{array}{c}10 \\
9\end{array}$ & $\begin{array}{c}10 \\
6 \\
\end{array}$ & $\begin{array}{c}10 \\
1\end{array}$ & $\begin{array}{c}10 \\
5\end{array}$ & $\begin{array}{c}12 \\
0\end{array}$ & $\begin{array}{c}10 \\
5\end{array}$ & $\begin{array}{c}12 \\
2 \\
\end{array}$ & 98 & $\begin{array}{c}10 \\
4 \\
\end{array}$ & 112 & 100 & $\begin{array}{c}10 \\
6 \\
\end{array}$ & 10 & 89 & 122 \\
\hline 4 & Altura brazo estirado & $\begin{array}{c}21 \\
7\end{array}$ & $\begin{array}{c}22 \\
0\end{array}$ & $\begin{array}{c}21 \\
6\end{array}$ & $\begin{array}{c}20 \\
0\end{array}$ & $\begin{array}{c}20 \\
4\end{array}$ & $\begin{array}{c}21 \\
8\end{array}$ & $\begin{array}{c}14 \\
7 \\
\end{array}$ & $\begin{array}{c}21 \\
6\end{array}$ & $\begin{array}{c}20 \\
0\end{array}$ & $\begin{array}{c}1 \\
20 \\
1\end{array}$ & 215 & 198 & $\begin{array}{c}20 \\
4\end{array}$ & 20 & 172 & 237 \\
\hline 5 & $\begin{array}{l}\text { Largo desde la vertical } \\
\text { al puño }\end{array}$ & 82 & 73 & 78 & 76 & 75 & 81 & 67 & 76 & 74 & 75 & 76 & 74 & 76 & 4 & 69 & 82 \\
\hline 6 & $\begin{array}{l}\text { Largo desde la vertical } \\
\text { al pecho }\end{array}$ & 26 & 24 & 40 & 20 & 24 & 25 & 23 & 24 & 16 & 21 & 23 & 20 & 24 & 6 & 14 & 33 \\
\hline 7 & Anchura de hombros & 43 & 43 & 59 & 46 & 44 & 45 & 43 & 43 & 42 & 48 & 46 & 46 & 46 & 5 & 38 & 53 \\
\hline 8 & Ancho de clavículas & 38 & 33 & 40 & 35 & 36 & 36 & 29 & 20 & 36 & 36 & 41 & 39 & 35 & 6 & 26 & 44 \\
\hline 9 & $\begin{array}{l}\text { Altura del glúteo al } \\
\text { arco de media espalda }\end{array}$ & 21 & 23 & 25 & 25 & 34 & 29 & 27 & 27 & 56 & 60 & 56 & 58 & 37 & 16 & 11 & 62 \\
\hline 10 & $\begin{array}{l}\text { Altura del piso a la } \\
\text { mitad de la espinilla }\end{array}$ & 38 & 45 & 43 & 39 & 42 & 48 & 43 & 47 & 41 & 40 & 46 & 40 & 43 & 3 & 37 & 48 \\
\hline 11 & $\begin{array}{l}\text { Largo desde el glúteo a } \\
\text { la rodilla }\end{array}$ & 53 & 56 & 57 & 48 & 64 & 57 & 55 & 59 & 42 & 44 & 46 & 44 & 52 & 7 & 40 & 64 \\
\hline 12 & Anchura de hombros & 51 & 42 & 60 & 47 & 41 & 45 & $\begin{array}{l}4 \\
0\end{array}$ & 49 & 14 & 14 & 17 & 15 & 36 & 17 & 9 & 63 \\
\hline 13 & $\begin{array}{l}\text { Largo de la mano al } \\
\text { dedo medio }\end{array}$ & 19 & 18 & 15 & 16 & 18 & 18 & 17 & 14 & 19 & 20 & 22 & 18 & 18 & 2 & 14 & 21 \\
\hline 14 & Anchura de mano & 10 & 11 & 12 & 10 & 12 & 10 & 11 & 10 & 17 & 14 & 17 & 19 & 13 & 3 & 7 & 18 \\
\hline 15 & Anchura de muñeca & 8 & 7 & 10 & 9 & 8 & 7 & 6 & 6 & 10 & 10 & 11 & 11 & 9 & 2 & 6 & 12 \\
\hline 16 & Diámetro de agarre & 4 & 3 & 3 & 4 & 4 & 4 & 3 & 6 & 6 & 7 & 6 & 6 & 5 & 1 & 2 & 7 \\
\hline 17 & Largo del pie & 27 & 27 & 28 & 25 & 28 & 30 & 25 & 29 & 4 & 3 & 4 & 3 & 19 & 12 & o & 39 \\
\hline 18 & Anchura del pie & 11 & 10 & 11 & 10 & 10 & 10 & 9 & 11 & 27 & 27 & 28 & 26 & 16 & 8 & 2 & 29 \\
\hline 19 & Altura en cuclillas & $\begin{array}{c}12 \\
9\end{array}$ & $\begin{array}{c}12 \\
0\end{array}$ & $\begin{array}{c}12 \\
9\end{array}$ & $\begin{array}{c}11 \\
5\end{array}$ & $\begin{array}{l}13 \\
0\end{array}$ & $\begin{array}{c}13 \\
7 \\
\end{array}$ & $\begin{array}{c}12 \\
3\end{array}$ & 121 & 116 & 124 & 125 & 124 & $\begin{array}{c}12 \\
4\end{array}$ & 6 & 114 & 135 \\
\hline 20 & $\begin{array}{l}\text { Altura del piso a brazo } \\
\text { extendido }\end{array}$ & $\begin{array}{c}15 \\
4 \\
4\end{array}$ & $\begin{array}{c}14 \\
9\end{array}$ & $\begin{array}{c}14 \\
4 \\
4\end{array}$ & $\begin{array}{c}14 \\
7 \\
\end{array}$ & $\begin{array}{c}14 \\
8\end{array}$ & $\begin{array}{c}14 \\
7\end{array}$ & $\begin{array}{c}14 \\
5\end{array}$ & $\begin{array}{c}14 \\
6 \\
\end{array}$ & 137 & 142 & $\begin{array}{c}14 \\
3\end{array}$ & 136 & $\begin{array}{c}14 \\
5\end{array}$ & 5 & 137 & 153 \\
\hline 21 & Edad & 26 & $2 O$ & 33 & 25 & 56 & 43 & 27 & 22 & 36 & 19 & 23 & 29 & 30 & 11 & 12 & 48 \\
\hline 22 & Peso en $\mathrm{kg}$ & 88 & 90 & 79 & 72 & 87 & 70 & $\begin{array}{l}6 \\
8 \\
\end{array}$ & 85 & 72 & 67 & 80 & 70 & 77 & 8 & 63 & 91 \\
\hline
\end{tabular}

Nota. P5: percentil 5; P95: percentil 95.

En la tabla 7 se observan intervalos muy amplios por lo que el riesgo para un sector de trabajadores es permanente debido a que las estaciones de trabajo no son flexibles ni ajustables, aun realizando consideraciones en diseños por percentiles o por cuartiles. 
Tabla 7. Percentiles de los 16 trabajadores con todas las variables.

\begin{tabular}{|c|c|c|c|c|c|}
\hline Medidas & $\mathbf{N}$ & Percentil 50 & $\begin{array}{c}\text { Desviación } \\
\text { típica }\end{array}$ & Percentil 5 & Percentil 95 \\
\hline Altura del cuerpo & 16 & 172,19 & 8,068 & 156 & 184 \\
\hline Altura al hombro & 16 & 148,81 & 6,575 & 138 & 160 \\
\hline Altura al codo & 16 & 106,44 & 8,907 & 84 & 122 \\
\hline Altura brazo estirado & 16 & 206,00 & 17,870 & 147 & 222 \\
\hline $\begin{array}{l}\text { Largo desde la vertical al } \\
\text { puño }\end{array}$ & 16 & 75,44 & 3,932 & 67 & 82 \\
\hline $\begin{array}{l}\text { Largo desde la vertical al } \\
\text { pecho }\end{array}$ & 16 & 23,56 & 5,072 & 16 & 40 \\
\hline Anchura de hombros & 16 & 45,19 & 4,053 & 42 & 59 \\
\hline Ancho de clavículas & 16 & 34,19 & 5,492 & 20 & 41 \\
\hline $\begin{array}{l}\text { Altura del glúteo al arco } \\
\text { de media espalda }\end{array}$ & 16 & 36,00 & 15,603 & 21 & 60 \\
\hline $\begin{array}{l}\text { Altura del piso a la mitad } \\
\text { de la espinilla }\end{array}$ & 16 & 43,13 & 2,964 & 38 & 48 \\
\hline $\begin{array}{l}\text { Largo desde el glúteo a la } \\
\text { rodilla }\end{array}$ & 16 & 52,50 & 6,429 & 42 & 64 \\
\hline Anchura de hombros & 16 & 36,63 & 15,747 & 14 & 60 \\
\hline $\begin{array}{l}\text { Largo de la mano al dedo } \\
\text { medio }\end{array}$ & 16 & 17,69 & 1,957 & 14 & 22 \\
\hline Anchura de mano & 16 & 12,63 & 3,117 & 10 & 19 \\
\hline Anchura de muñeca & 16 & 8,38 & 1,784 & 6 & 11 \\
\hline Diámetro de agarre & 16 & 4,69 & 1,448 & 3 & 7 \\
\hline Largo del pie & 16 & 19,75 & 11,192 & 3 & 30 \\
\hline Anchura del pie & 16 & 15,50 & 8,327 & 9 & 29 \\
\hline Altura en cuclillas & 16 & 122,81 & 6,685 & 109 & 137 \\
\hline $\begin{array}{l}\text { Altura del piso a brazo } \\
\text { extendido }\end{array}$ & 16 & 145,13 & 4,380 & 136 & 154 \\
\hline $\begin{array}{l}\text { MD_Dedo pulgar - dedo } \\
\text { índice }\end{array}$ & 16 & 85,75 & 7,946 & 60 & 95 \\
\hline $\begin{array}{l}\text { MD_Dedo índice - dedo } \\
\text { medio }\end{array}$ & 16 & 52,38 & 8,358 & 44 & 73 \\
\hline $\begin{array}{l}\text { MD_Dedo medio - dedo } \\
\text { anular }\end{array}$ & 16 & 45,13 & 11,558 & 34 & 64 \\
\hline $\begin{array}{l}\text { MD_Dedo anular - dedo } \\
\text { meñique }\end{array}$ & 16 & 45,31 & 14,351 & 31 & 70 \\
\hline MD_Abducción & 16 & 91,56 & 7,519 & 72 & 98 \\
\hline MD_Supinación & 16 & 50,06 & 11,174 & 35 & 67 \\
\hline MD_Lateral izquierdo & 16 & 74,13 & 23,255 & 39 & 128 \\
\hline MD_Lateral derecho & 16 & 60,13 & 14,472 & 38 & 104 \\
\hline $\begin{array}{l}\text { MI_Dedo pulgar - dedo } \\
\text { índice }\end{array}$ & 16 & 84,94 & 6,990 & 68 & 94 \\
\hline $\begin{array}{l}\text { MI_Dedo índice - dedo } \\
\text { medio }\end{array}$ & 16 & 51,19 & 7,064 & 42 & 63 \\
\hline $\begin{array}{l}\text { MI_Dedo medio - dedo } \\
\text { anular }\end{array}$ & 16 & 44,31 & 9,492 & 32 & 57 \\
\hline MI_Abducción & 16 & 85,69 & 11,116 & 57 & 97 \\
\hline $\begin{array}{l}\text { MI_Dedo anular - dedo } \\
\text { meñique }\end{array}$ & 16 & 45,06 & 10,661 & 34 & 63 \\
\hline
\end{tabular}

El análisis de contingencia se observa en la primera parte de matriz de análisis estadístico en la tabla 8. 
Tabla 8. Tabla de contingencia, primera parte.

\begin{tabular}{|c|c|c|c|c|c|c|c|c|c|c|c|c|c|c|c|c|c|c|c|}
\hline & 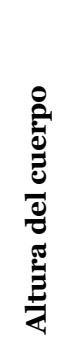 & 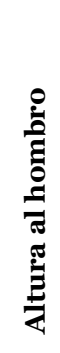 & 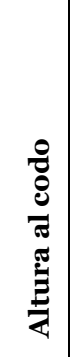 & 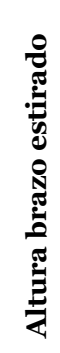 & 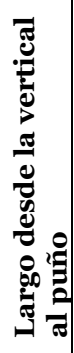 & 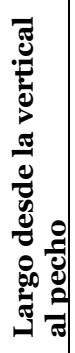 & 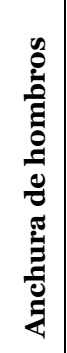 & 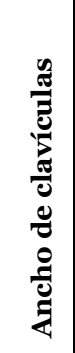 & 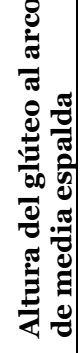 & 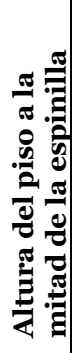 & 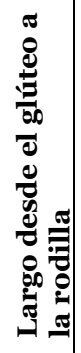 & 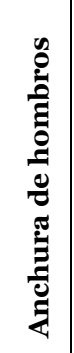 & 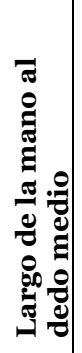 & 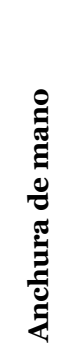 & 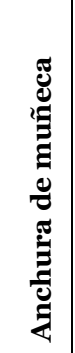 & 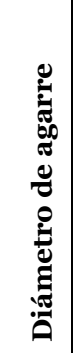 & 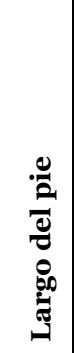 & 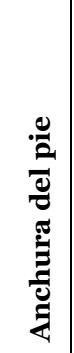 & 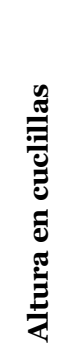 \\
\hline $\begin{array}{c}\text { Chi- } \\
\text { cuadrado }\end{array}$ & $\begin{array}{c}3,5 \\
\text { oo } \\
\text { a }\end{array}$ & $\begin{array}{c}1,5 \\
\text { oo } \\
\mathrm{c}\end{array}$ & $\begin{array}{c}1,5 \\
\text { oo } \\
\mathrm{c}\end{array}$ & $\begin{array}{l}1,8 \\
75^{\mathrm{a}} \\
\end{array}$ & $\begin{array}{l}3,2 \\
50^{d}\end{array}$ & $\begin{array}{l}5,3 \\
75^{\mathrm{e}}\end{array}$ & $\begin{array}{l}5,8 \\
75^{f}\end{array}$ & $\begin{array}{l}\text { 6,o } \\
\text { oo }^{d}\end{array}$ & $\begin{array}{l}3,2,2 \\
5 \mathrm{O}^{\mathrm{d}}\end{array}$ & $\begin{array}{l}3,2 \\
50^{d}\end{array}$ & $\begin{array}{c}1,5 \\
\text { oo } \\
\mathrm{g}\end{array}$ & $\begin{array}{l}2, \mathrm{O} \\
\mathrm{OO}^{\mathrm{h}}\end{array}$ & $\begin{array}{l}5,0 \\
0^{i}\end{array}$ & $\begin{array}{l}5,0 \\
\text { ooj }\end{array}$ & $\begin{array}{l}2,0 \\
\text { ooj }\end{array}$ & $\begin{array}{l}3,3 \\
75^{k}\end{array}$ & $\begin{array}{l}2,0 \\
0^{e}\end{array}$ & $\begin{array}{l}8,5 \\
\text { oo }^{f}\end{array}$ & $\begin{array}{l}1,8 \\
75^{\mathrm{a}}\end{array}$ \\
\hline gl & 12 & 13 & 13 & 12 & 10 & 8 & 6 & 10 & 10 & 10 & 9 & 11 & 7 & 5 & 5 & 4 & 8 & 6 & 12 \\
\hline
\end{tabular}

Nota. a. 13 casillas $(100,0 \%)$ tienen frecuencias esperadas menores que 5 . La frecuencia de casilla esperada mínima es 1,2 ; b. 14 casillas $(100,0 \%)$ tienen frecuencias esperadas menores que 5 . La frecuencia de casilla esperada mínima es 1,1 ; c. 11 casillas $(100,0 \%)$ tienen frecuencias esperadas menores que 5. La frecuencia de casilla esperada mínima es 1,5; d. 9 casillas $(100,0 \%)$ tienen frecuencias esperadas menores que 5 . La frecuencia de casilla esperada mínima es 1,8; e. 7 casillas $(100,0 \%)$ tienen frecuencias esperadas menores que 5 . La frecuencia de casilla esperada mínima es 2,3 .

Las de menor coincidencia son: diámetro de agarre, anchura de muñeca, anchura de mano, es decir, todos los operarios tienen que trabajar con las mismas dimensiones de mangos de herramientas, aun cuando sus manos tengan diferencias muy amplias en dimensiones, con lo cual se puede inferir que las manos y dedos están expuestos a riesgos ergonómicos.

La segunda parte de matriz de análisis estadístico se muestra en la tabla 9.

Tabla 9. Tabla de contingencia, segunda parte.

\begin{tabular}{|c|c|c|c|c|c|c|c|c|c|c|c|c|c|c|c|c|c|c|c|}
\hline & 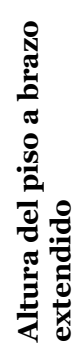 & 胥 & 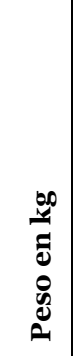 & 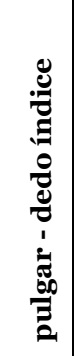 & 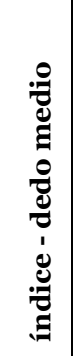 & 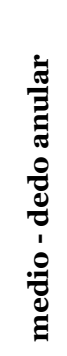 & 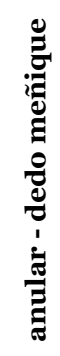 & 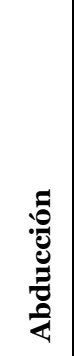 & 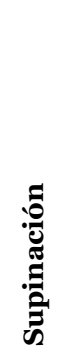 & 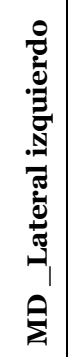 & 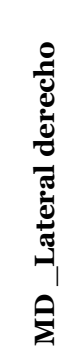 & 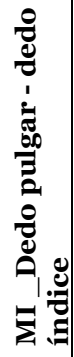 & 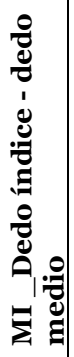 & 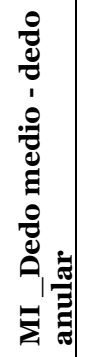 & 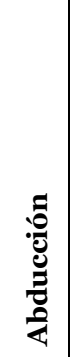 & 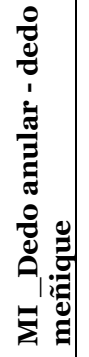 & 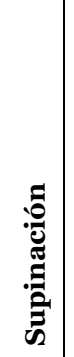 & 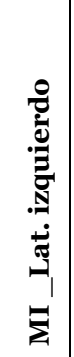 & 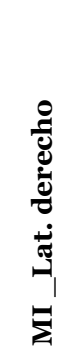 \\
\hline $\begin{array}{c}\text { Chi- } \\
\text { cuadrado }\end{array}$ & $\begin{array}{l}3,2 \\
50^{d}\end{array}$ & $\begin{array}{c}1,5 \\
\text { oO } \\
\text { c }\end{array}$ & $\begin{array}{l}3,2 \\
50^{\mathrm{d}}\end{array}$ & $\begin{array}{l}3,2 \\
50^{\mathrm{d}}\end{array}$ & $\begin{array}{l}6,5 \\
\text { oog }^{2}\end{array}$ & $\begin{array}{l}1,8 \\
75^{\mathrm{d}}\end{array}$ & $\begin{array}{l}1,8 \\
75^{\mathrm{a}}\end{array}$ & $\begin{array}{l}4,0 \\
\text { oog }\end{array}$ & $\begin{array}{c}1,5 \\
\text { oo } \\
c\end{array}$ & $\begin{array}{l}1,8 \\
75^{\mathrm{a}}\end{array}$ & $\begin{array}{l}2, \mathrm{O} \\
\mathrm{OO}^{\mathrm{h}}\end{array}$ & $\begin{array}{l}1,8 \\
75^{\mathrm{a}}\end{array}$ & $\begin{array}{l}1,8 \\
75^{\mathrm{a}}\end{array}$ & $\begin{array}{l}1,8 \\
75^{\mathrm{d}}\end{array}$ & $\begin{array}{c}3,5 \\
\text { oo } \\
\mathrm{h}\end{array}$ & $\begin{array}{l}3,5 \\
\text { oO }^{\mathrm{a}}\end{array}$ & $\begin{array}{l}1,8 \\
75^{\mathrm{a}}\end{array}$ & $\begin{array}{c}1,5 \\
\text { oo } \\
\text { c }\end{array}$ & $\begin{array}{l}4,0 \\
\text { oog }\end{array}$ \\
\hline gl & 10 & 13 & 10 & 10 & 9 & 10 & 12 & 9 & 13 & 12 & 11 & 12 & 12 & 10 & 11 & 12 & 12 & 13 & 9 \\
\hline
\end{tabular}

Nota. f. 10 casillas $(100,0 \%)$ tienen frecuencias esperadas menores que 5 . La frecuencia de casilla esperada mínima es 1,6; g. 12 casillas $(100,0 \%)$ tienen frecuencias esperadas menores que 5 . La frecuencia de casilla esperada mínima es 1,3 ; h. 8 casillas $(100,0 \%)$ tienen frecuencias esperadas menores que 5. La frecuencia de casilla esperada mínima es 2,0; i. 6 casillas (100,0\%) tienen frecuencias esperadas menores que 5 . La frecuencia de casilla esperada mínima es 2,7; j. 5 casillas $(100,0 \%)$ tienen frecuencias esperadas menores que 5 . La frecuencia de casilla esperada mínima es 3,2 . 
En la tabla 9 destaca la baja frecuencia en dimensionamiento del ángulo entre el dedo pulgar y dedo índice, así como entre el dedo índice y el dedo medio; factores relevantes para el levantamiento de masas, uso de herramientas y agarre adecuado. De los puntos primordiales, cabe destacar ampliamente las dimensiones de las manos, tales como la longitud de la palma, ancho palma, y diámetro de agarre entre otros, debido a que el análisis muestra mayores puntos críticos y de variabilidad.

Esto llama la atención debido al uso de herramientas por el tipo de trabajo y el agarre al hacer los levantamientos; de alguna manera se puede inferir que existe alguna relación entre las dimensiones de las manos y las lesiones que han sufrido los trabajadores, además de las posturas inadecuadas y los pesos excesivos que levantan.

$\mathrm{Al}$ analizar la estación de trabajo se encontró que el peso de las piezas está entre 45 y 50 $\mathrm{kg}$, con lo que supera los $25 \mathrm{~kg}$ que marca la Norma Oficial Mexicana NOM-o06-STPS-2014 para un operario, sin la ayuda de otra herramienta de carga. Mientras se levanta la carga el tronco se flexiona, retuerce y se dobla hacia un lado, con un nivel de riesgo rojo.

Figura 5. Estación de trabajo.

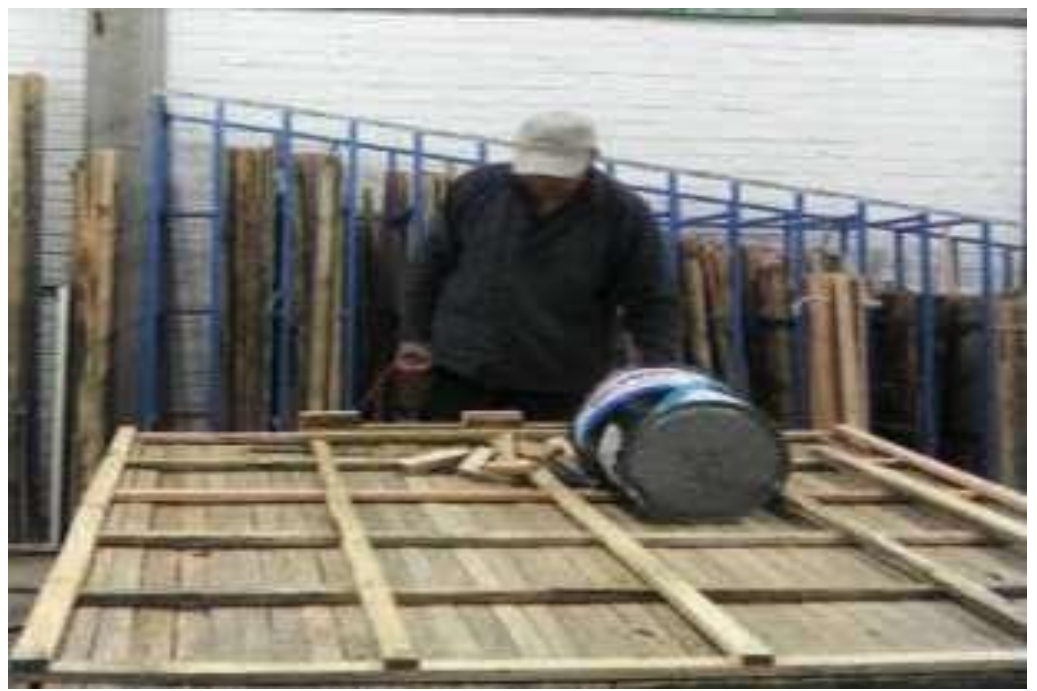

\section{Conclusiones}

De la aplicación del método Suzanne Rodgers para evaluar las posturas, fuerzas y actividad muscular, se evidencia riesgo relacionado con adquirir DTA's por fatiga acumulada en seis de las nueve variables asociadas con las condiciones de trabajo. En lo referente al estudio antropométrico, se pudo comprobar la diversificación de los diseños de los lugares de trabajo, equipos y herramientas, con respecto a las características de los trabajadores y aún entre ellos mismos y las actividades que desarrollan, los cuales no corresponden a las dimensiones de los usuarios y esto ocasiona que se lastimen constantemente, en especial con el tamaño de las herramientas.

De los puntos primordiales, cabe destacar la importancia de las dimensiones de las manos, tales como la longitud de la palma, ancho palma, y diámetro de agarre entre otros, 
debido a que el análisis muestra mayores puntos críticos y de variabilidad. Esto llama la atención debido al uso de herramientas por el tipo de trabajo y el agarre al hacer los levantamientos; de alguna manera se puede inferir que existe alguna relación entre las dimensiones de las manos y las lesiones que han sufrido los trabajadores, además de las posturas inadecuadas y los pesos excesivos que levantan; por lo cual se llevarán a cabo otros estudios a futuro con una muestra mayor y en otras áreas de producción para realizar un comparativo.

Se propuso buscar otras herramientas y métodos de prueba o materiales alternativos acordes con las dimensiones antropométricas de las personas que las utilizan dado que, se encontró riesgo alto de adquirir DTA's debido a las dimensiones de las herramientas no compatibles con dichas medidas que causan una mayor fatiga y lesiones a los usuarios, y en el peor de los casos, accidentes de trabajo y consecuencias fatales para el trabajador. Las tablas muestran como las diferencias individuales pueden ser muy grandes, como las de constitución física y fuerza, que son evidentes, pero hay otras, como las diferencias culturales, de estilo de habilidades que son más difíciles de identificar por lo que se requieren estudios más exhaustivos.

En el análisis sobre los procesos se pudo apreciar que los trabajadores realizan levantamientos de objetos entre 45 y $50 \mathrm{~kg}$ sin ayuda mecánica, mayores a $25 \mathrm{~kg}$, que marca la Norma Oficial Mexicana NOM-036-1-STPS-2018 como límite máximo. Dentro de los movimientos peligrosos también se perciben giros bruscos e inesperados, posturas antinaturales continuas, extensiones y levantamientos. Además de que los procedimientos no se enfocan a las actividades de empaque, solo se llevan a cabo en áreas de maquinado; es decir, los empacadores realizan las actividades de manera empírica y sin ninguna capacitación.

Los trastornos musculoesqueléticos no sólo representan un problema para las empresas, sino también para el Estado, pues el gobierno tiene que destinar más recursos para atender estas lesiones. La categoría de trabajadores mayores varía según su condición funcional, que a su vez está influenciada por su historial laboral previo. Depende también del trabajo que ocupen y de su situación social, cultural y económica del lugar donde viven. Los trabajadores que realizan un trabajo puramente físico suelen ser los que tienen el menor nivel de escolaridad y preparación profesional, están sujetos al estrés causado por el trabajo extenuante, que puede ser una causa de enfermedad, y expuestos al riesgo de accidentes laborales. En este contexto, es más que probable que su capacidad física disminuya al final de su vida activa, hecho que los convierte en trabajadores más vulnerables.

Para realizar las actividades de carga, los centros de trabajo deben contar con un procedimiento que incluya la descripción de la técnica adecuada para realizar las tareas de manera segura; las medidas de seguridad y control que se aplican durante el transcurso del trabajo; las características de la carga tales como dimensiones, forma o peso; condiciones ambientales que pueden incrementar el esfuerzo laboral; la trayectoria para la transferencia de la carga, y la especificación de los materiales que se manipulan.

Se concluye que la tarea de empaque de muebles de oficina, es una actividad donde existe alto riesgo de adquirir DTA`s y que hay relación entre las medidas antropométricas de 
los trabajadores y los accidentes de trabajo, específicamente en el uso de herramientas manuales.

Se recomienda a futuro realizar un estudio integral a largo plazo, una reestructuración del lugar de trabajo a mediano plazo y la formación de supervisores y trabajadores para concientizar sobre los riesgos y la importancia de cuidar los riesgos laborales, en el corto plazo; es decir, es necesario adoptar un enfoque sistémico urgentemente con un cambio de mentalidad de los líderes. Está claro que los beneficios de la ergonomía pueden reflejarse de muchas formas diferentes: en productividad y calidad, en seguridad y salud, en confiabilidad, en satisfacción laboral y en desarrollo personal; sin embargo, la más importante de todas ellas es la de preservar la vida. 


\section{Referencias}

Apud, E. \& Meyer, F. (2003). La importancia de la ergonomía para los profesionales de la salud. Ciencia y Enfermería, 9(1), 15-20. http://dx.doi.org/10.4067/So717-95532003000100003

Ávila, R., Prado, L. R., \& González, E. L. (2007). Dimensiones Antropométricas de Población Latinoamericana. Universidad de Guadalajara.

Cornejo, R. \& Quiñónez, M. (2007). Factores asociados al malestar/bienestar docente. Una investigación actual. Revista Iberoamericana sobre Calidad, Eficacia y Cambio en Educación, 5(5) 75-80. https://www.redalyc.org/pdf/551/55121025011.pdf

Diario Oficial de la Federación (2020). Reglamento Federal de Seguridad y Salud en el trabajo. $\begin{array}{lllllll}\text { Consultado el } & 3 & \text { de } & \text { marzo }\end{array}$ http://www.dof.gob.mx/nota detalle.php?codigo $=5368114 \&$ fecha $=13 / 02 / 2020$

Galvis, J. F., Pérez, J. M., \& Ramírez, E. (2015). Carga física en trabajadores del área de acabados en industria metalmecánica. Revista Colombiana de Salud Ocupacional, 5(4), 23-26. https://doi.org/10.18041/2322-634X/rcso.4.2015.4932

González, J. $\quad$ L. $\quad$ (2007). Lesiones musculoesqueléticas. http://www.ergocupacional.com/4910/57873.html

Grandjean, E. (1983). Précis d'ergonomie. Editions d'Organisation.

Hendrick, H. (1991). Macroergonomics: A better approach to work system design. Revista IEA, 11, 76-81.

Hertzberg, H. T. (1968). The conference on standardization of anthropometric techniques and terminology. American Journal of Physical Anthropology, 28(1), 1-16. https://doi.org/10.1002/ajpa.1330280110

International Ergonomics Association (2016). Clasificación de la ergonomía en la asociación internacional de ergonomía. Consultado el 20 de diciembre de 2016 de Sitio web: www.iea.cc/whats/index.html

Marín-Zurdo, J. J., Boné-Pina, M. J., \& Benito-Gil, C. (2013). Evaluación de riesgos de manipulación repetitiva a alta frecuencia basada en análisis de esfuerzos dinámicos en las articulaciones sobre modelos humanos digitales. Ciencia \& Trabajo, 15(47), 86-93. https://dx.doi.org/10.4067/S0718-24492013000200009

Mondelo, P. R., Gregori, E., \& Barrau, P. (1999). Ergonomía 1 Fundamentos. Ediciones de la Universidad Politécnica de Catalunya.

Norma Oficial Mexicana NOM-o06-STPS-2014. Manejo y almacenamiento de materialesCondiciones de seguridad y salud en el trabajo. Diario Oficial de la Federación, Ciudad de México, México, 11 de septiembre de 2014.

Norma Oficial Mexicana NOM-036-1-STPS-2018. Factores de riesgo ergonómico en el TrabajoIdentificación, análisis, prevención y control. Parte 1: Manejo manual de cargas. Diario Oficial de la Federación, Ciudad de México, México, 23 de noviembre de 2018.

Organización Internacional del Trabajo (2013). La prevención de enfermedades profesionales. OIT. https://www.ilo.org/safework/info/WCMS 2095.55/lang--es/index.htm

Universidad Politécnica de Valencia (2006). Métodos de evaluación de la ergonomía de puestos de trabajo. Ergonautas. https://www.ergonautas.upv.es/metodos-evaluacionergonomica.html 